\title{
Universal Method for Extracting Transport Parameters From Monte Carlo Device Simulation
}

\author{
Simon C. Brugger and Andreas Schenk
}

\begin{abstract}
The extraction of transport parameters as mobilities and diffusivities has been a subject of research for more than 20 years. However, the solutions proposed up to now are not satisfactory particularly when applied to nanoscale devices. In this brief, we review the two most popular methods to extract mobilities and show how they fail in nanoscale devices, where transport is strongly quasi-ballistic. We also show that these methods are not appropriate to extract tensorial transport parameters. As an alternative, we propose to use recently derived general definitions of mobilities and diffusivities that naturally solve these problems and, thus, constitute a universal method to extract transport parameters.
\end{abstract}

Index Terms-Boltzmann equation, device simulation, generalized drift-diffusion equation, inverse scattering operator, transport parameters.

\section{INTRODUCTION}

$\mathbf{T}$ HE IDEA to extract transport parameters from a Monte Carlo (MC) solution of the Boltzmann equation and to insert them in a generalized drift-diffusion equation is not new [1], [2]. It has been proposed by many authors as a promising method to take into account hot-electron effects, as well as generation-recombination, in a self-consistent way [1], [3], [4]. This method has, however, never found a wide domain of application. In this brief, we argue that the proposed methods for extracting transport parameters are afflicted with four major problems. As we will show, these problems are all related to the previously proposed extraction methods, which are based on the usage of the mean particle velocity to define mobility. After carefully describing these four issues in Section II, we will show in Section III that, with proper definitions of the transport parameters based on the exact theory of the inverse scattering operator [5], all these problems are naturally resolved. Conclusions will be given in the last section.

Manuscript received January 19, 2007; revised July 3, 2007. This work was supported in part by the Swiss National Science Foundation (project NEQUATTRO SNF 200021-109393/1) and in part by the European project PULLNANO (IST-4-026828). The review of this brief was arranged by Editor C. McAndrew.

S. C. Brugger is with the Integrated Systems Laboratory, Swiss Federal Institute of Technology (ETH) Zürich, 8092 Zürich, Switzerland (e-mail: brugger@iis.ee.ethz.ch).

A. Schenk is with the Integrated Systems Laboratory, Swiss Federal Institute of Technology (ETH) Zürich, 8092 Zürich, Switzerland, and also with the Synopsys LLC, 8050 Zürich, Switzerland.

Color versions of one or more of the figures in this brief are available online at http://ieeexplore.iee.org.

Digital Object Identifier 10.1109/TED.2007.906937

\section{Problems of Previous Extraction Methods}

In [1], Park et al. defined the local electron mobility $\mu_{i i}$ in the transport direction $i$ as

$$
\mu_{i i}:=-\frac{\left\langle v_{i}\right\rangle_{\mathrm{MC}}}{\partial_{i} \psi_{n}}
$$

where $\left\langle v_{i}\right\rangle_{\mathrm{MC}}$ is the mean electron velocity in the transport direction $i$ as computed with the MC method, and $\partial_{i} \psi_{n}$ is the derivative of the quasi-Fermi potential in the transport direction $i$. Here, the mean value $\langle g\rangle$ of a function $g$ is defined using the bra and ket notation as in [5]

$$
\langle g\rangle:=\frac{\langle g \mid f\rangle}{\langle 1 \mid f\rangle}=\frac{\langle g \mid f\rangle}{n}
$$

where $f$ is the solution of the Boltzmann equation, and $n:=\langle 1 \mid f\rangle$ is the density. Note that (1) is strictly valid as long as the Einstein relation holds. The proof of this statement is given in the Appendix. Definition (1) involves two of the four problems that we would like to discuss. First, since the velocity $\vec{v}(\vec{k})$ is an odd function of the quasi-momentum $\vec{k}$, it is difficult to compute efficiently using a MC method as already pointed out in [1]. Second, the velocity, as well as the gradient of the quasi-Fermi potential, is zero perpendicular to the current flow direction, making it impossible to define a proper mobility perpendicular to the transport direction.

To obtain a better definition of the mobility, which also holds far from equilibrium, Bandyopadhyay et al. [3] derived a generalized expression for $\mu_{i j}$ as

$$
\mu_{i j}:=\frac{q\left\langle v_{i}\right\rangle_{\mathrm{MC}}}{\left\langle\left(\frac{d p_{j}}{d t}\right)_{\mathrm{coll}}\right\rangle_{\mathrm{MC}}}
$$

where $\left\langle\left(d p_{j} / d t\right)_{\text {coll }}\right\rangle_{\mathrm{MC}}$ is the mean value of the rate of loss of electron momentum due to collisions in the $j$ th direction. Unfortunately, this method generates even more problems than the first one, even though it should be valid far from equilibrium.

\section{A. Odd Moments}

The first problem is the same as the previous discussion. For a better understanding, the denominator of (3) is rewritten using 


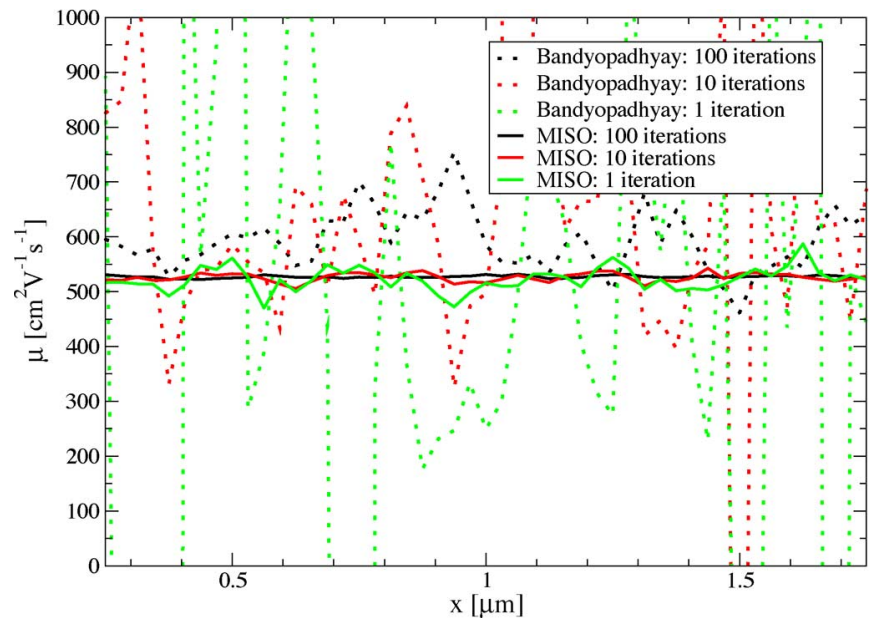

Fig. 1. Mobility in the transport direction as a function of the position and of the number of iterations.

the notation of [5]

$$
\left\langle\left(\frac{d p_{i}}{d t}\right)_{\mathrm{coll}}\right\rangle_{\mathrm{MC}}:=\frac{\hbar\left\langle k_{i}|S| f_{\mathrm{MC}}\right\rangle}{\left\langle 1 \mid f_{\mathrm{MC}}\right\rangle} .
$$

As the function $\left[\left\langle k_{i}\right| S\right](\vec{k})$ is an odd function like $\vec{v}(\vec{k})$, its computation is also laborious when using the MC method. To illustrate this problem, a simple 2- $\mu$ m-long resistor has been simulated using the MC method. The doping concentration is $10^{17} \mathrm{~cm}^{-3}$, the electric field inside the device is $10 \mathrm{kV} / \mathrm{cm}$, and the crystallographic orientation is $\langle 110\rangle$. Fig. 1 shows the value of the local mobility as a function of the position after 1, 10, and 100 iterations, each iteration corresponding to a simulation time of $0.05 \mu \mathrm{s}$. The dashed lines are the results as obtained using the method by Bandyopadhyay et al. As expected, the results are very noisy since the mobility is a quotient of two odd moments, and a total simulation time of $5 \mu \mathrm{s}$ is not even enough to achieve a precision better than 5\%. This is a major drawback of the method.

As pointed out in [3], (4) can be rewritten as

$$
\left\langle\left(\frac{d p_{i}}{d t}\right)_{\mathrm{coll}}\right\rangle_{\mathrm{MC}}=\frac{-1}{n_{\mathrm{MC}}} \sum_{l} \partial_{l}\left(2 n_{\mathrm{MC}} u_{i l}\right)+q E_{i}
$$

where $E_{i}$ is the electric field in $i$-direction, $n_{\mathrm{MC}}$ is the MC density, and the definition of $u_{i l}$ is given in [3]. Expression (5) is not easier to compute than (4) due to the difficulty to compute the gradient of $n_{\mathrm{MC}}$ [4].

\section{B. Diagonal Terms Perpendicular to the Transport Direction}

In this section, we consider the transport parameters perpendicular to the transport direction. The determination of these parameters is important, among others, for small-signal analysis. For example, if one cannot determine the transport parameters perpendicular to the transport direction under the gate of a bulk MOSFET, the small-signal response to a small potential variation on the gate cannot be described properly.

By definition, the velocity perpendicular to the transport direction is zero; thus, the numerator of (3) becomes zero. What is

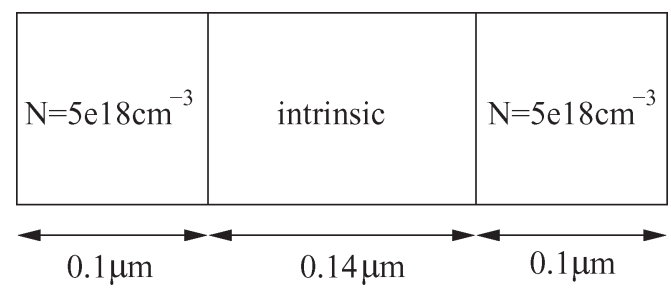

Fig. 2. Simple NIN structure where the Bandyopadhyay et al. definition of the mobility fails.

the consequence for the denominator of (3)? Considering, e.g., the line of the transport direction in the middle of a rectangular resistor, the denominator will also become zero because of mirror symmetry of the device. This leads to an undefined mobility perpendicular to the transport direction. One may argue that this only happens in cases of high symmetry, but, as the distribution function continuously depends on the geometry, the line will be transformed into a curve if one slightly deforms the device. Therefore, this kind of problem is expected in a large class of devices. In the case of a bulk MOSFET, the value of (4) perpendicular to the transport direction (i.e., perpendicular to the gate) will, in general, not be zero, leading to zero transverse mobilities almost everywhere. This is unphysical because it would lead to a vanishing gate conductance for nonvanishing frequencies, and the source-gate transconductance will also be affected because it partly depends on the transverse transport parameters. This is the second main drawback of the method proposed by Bandyopadhyay et al.

\section{Nondiagonal Terms}

Considering $i$ as the transport direction and $j(j \neq i)$ as a perpendicular direction, the nondiagonal term $\mu_{i j}$ of the mobility tensor will always diverge in a point with mirror symmetry in the $j$-direction because, in that case, the denominator is zero. The elements $\mu_{j i}$ will almost always vanish because the numerator is zero by definition, and sometimes, it will be undefined as we will see in the next section.

\section{Divergence in Transport Direction}

Sections II-B and C show that, using (3), the only component of the mobility tensor that seems to be always well defined is the component in transport direction. In this section, an example is described where the numerator of (3) is positive and the denominator is zero. Fig. 2 shows the schematic of a simple $N I N$ structure, where (3) fails even in the transport direction.

If the device shown in Fig. 2 is biased with $2 \mathrm{~V}$, the profile of the mean velocity [the numerator of (3)] turns out as shown in Fig. 3. The velocity is positive everywhere as expected. However, inspecting the denominator of (3) gives a somewhat different picture as one can see in Fig. 4. The mean rate of loss of momentum for the electrons is first positive, then crosses the $x$-axis to become negative, crosses the $x$-axis again, and is then positive. Using (3) leads to a mobility that diverges in two points in the transport direction. In addition, the nondiagonal terms with the transport direction as the second index are undefined $(0 / 0)$. The occurrence of a negative mobility between 


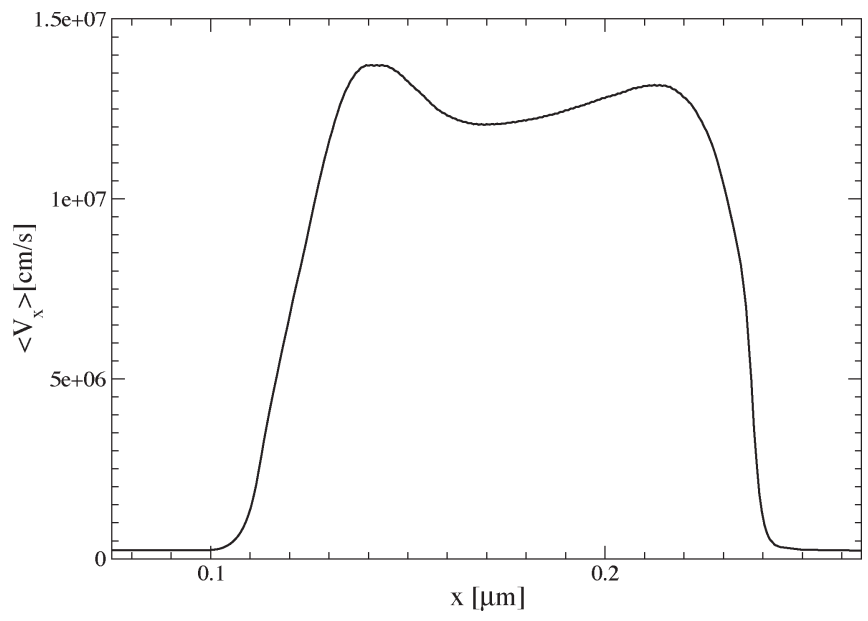

Fig. 3. Velocity profile at a bias of $2 \mathrm{~V}$.

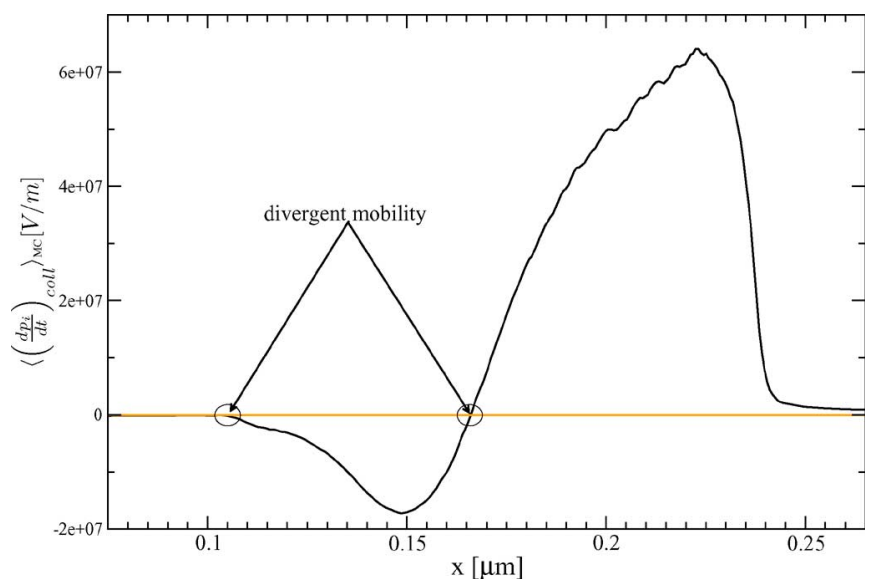

Fig. 4. Profile of the mean rate of loss of momentum for the electrons at a bias of $2 \mathrm{~V}$.

the two crossing points has already been investigated in [6]. With the method proposed by Bandyopadhyay et al., this case is not a singular one, but nanoscale MOSFETs, in general, are afflicted with this problem.

\section{UNIVERSAL METHOD}

The preceding section showed that the mobility extraction methods presented in [1] and [3] involve serious problems and are, therefore, inappropriate to extract transport parameters from the solution of the Boltzmann equation. This applies even more if they are used together with the MC method. In [7], Gan and Tang tried to solve the problem of the extraction of odd moments with MC (see Section II-A). Unfortunately, the method by Gan and Tang based on a spherical harmonics expansion of the distribution function is only valid in the effective mass approximation and as long as the distribution functions are not too asymmetrical.

In this section, we show that the method proposed in [5] to extract transport parameters solves all four problems at the same time without any restrictions to the subjacent Boltzmann equation (no more approximations than already contained in the Boltzmann equation itself).

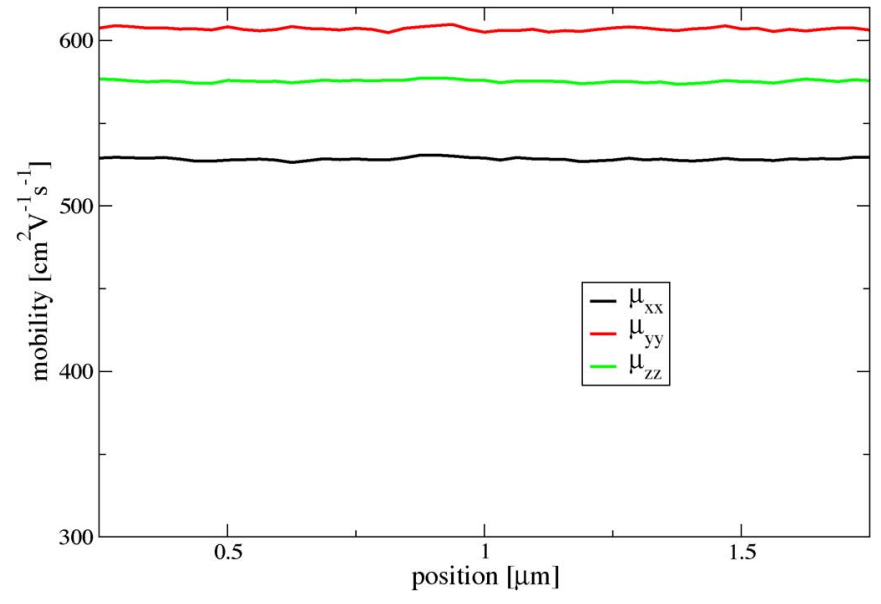

Fig. 5. Profile of the diagonal components of the mobility tensor for electrons in a simple resistor.

\section{A. Proper Definition of a Mobility Tensor}

In [5], the definition of the mobility tensor is based on the velocity moment of the inverse scattering operator (MISO) $S_{v_{i}}^{-1}$

$$
\mu_{i j}:=-\frac{q}{n \hbar} \int_{K} \partial_{k_{j}} S_{v_{i}}^{-1} f d^{3} k
$$

where $S$ is the scattering operator, and $v_{i}$ is the component of the velocity in $i$-direction. In [5], a mathematical proof was given that these moments always exist and that they are always computable.

\section{B. Advantages}

Contrary to (3), where the mobility was defined as a quotient of the mean values of odd functions, (6) defines the mobility as the mean value of an even function. This is crucial for the extraction using an MC method because mean values of even functions can be computed very efficiently. To illustrate this point, the mobility in the simple resistor of Section II-A was computed using (6). The plain lines in Fig. 1 show that our method produces more precise results after one iteration than the method by Bandyopadhyay et al. after 100 iterations. The derivative of the velocity MISO in front of the distribution function in (6) is computed once for all in a given scattering operator and a band structure (see [8]) and must therefore be considered as a known easily computable function.

The undefined or zero mobilities perpendicular to the transport direction as they occur with (3) are wiped off when using (6). Fig. 5 shows the diagonal components of the mobility tensor when the $x$-direction is the transport direction. All components are well defined and can be computed with the same accuracy. The difference between the $y$-direction (parallel to the device plane) and the $z$-direction (perpendicular to the device plane) results from the $\langle 110\rangle$ orientation of the silicon. The nondiagonal elements are all zero because of the symmetry of the system.

Fig. 6 shows that, using (6), the mobility in the $N I N$ structure does not diverge. The negative mobility has been investigated in [6]. 


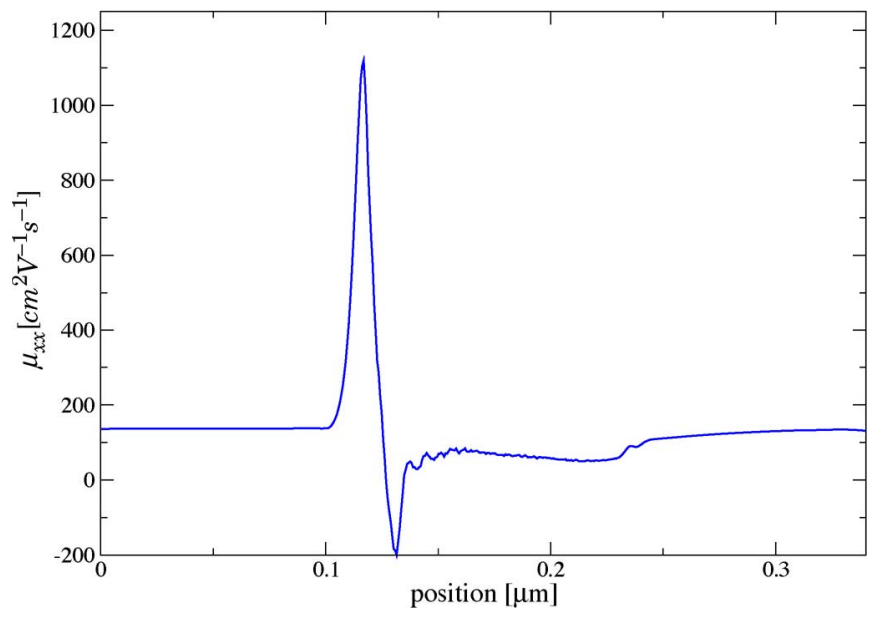

Fig. 6. Profile of the mobility component in the transport direction for electrons at a bias of $2 \mathrm{~V}$.

Everything outlined for the mobility tensor is likewise true for the diffusivity tensor.

\section{CONCLUSION}

The computation of the transport parameters based on the velocity moments of the inverse scattering operator [5] has the following advantages compared to other methods. First, the tensorial transport parameters are always well defined in the transport direction and, more importantly, perpendicular to it. The off-diagonal elements are also well defined. Second, as the mobility tensor and the diffusivity tensor are defined as the mean values of even functions, they are efficiently computable using the MC method.

In previous papers [9], [10], we have shown that those transport parameters can then really be inserted in a generalized drift-diffusion equation to accurately compute densities and current densities, and this independently of the working point. Although the idea of this method is not new, its realization only became possible because a proper (always valid and robust) definition of transport parameters has been derived.

\section{APPENDIX}

In this Appendix, we give a proof that the general definition of a mobility given in [5] coincides with (1) in the case of Boltzmann statistics for small gradients of the quasi-Fermi potential. The solution $f(\mathbf{r}, \mathbf{k})$ of the Boltzmann equation can always be written as

$$
f(\mathbf{r}, \mathbf{k})=: n(\mathbf{r}) g(\mathbf{r}, \mathbf{k})
$$

where $n(\mathbf{r})$ is the density, and $g(\mathbf{r}, \mathbf{k})$ has, by definition, the property

$$
\int_{K} g(\mathbf{r}, \mathbf{k}) d^{3} k=1 .
$$

The density can be rewritten as a function of the electrostatic potential $\phi$ and of the quasi-Fermi potential $\psi_{n}$ as

$$
n(\mathbf{r})=n_{i} \exp \left(\frac{q}{k_{\mathrm{B}} T}\left(\phi(\mathbf{r})-\psi_{n}(\mathbf{r})\right)\right)
$$

where $n_{i}$ is the intrinsic density, $q$ is the absolute value of the electron charge, $k_{\mathrm{B}}$ is the Boltzmann constant, and $T$ is the lattice temperature. Under the approximation

$$
g \nabla_{r} n \gg n \nabla_{r} g
$$

which is always fulfilled near thermodynamic equilibrium, the expression for the solution of the Boltzmann equation in the first order in the electric field and in the gradient of the quasiFermi potential can be written as (the derivation is identical to that given in [8, pp. 49-50])

$$
\begin{aligned}
f(\mathbf{r}, \mathbf{k})=f_{\mathrm{eq}}(\mathbf{r}, \mathbf{k})\left[1-\frac{q}{k_{\mathrm{B}} T} \nabla_{r} \psi_{n}(\mathbf{r}) \cdot S_{\vec{v}}^{-1}(\mathbf{r}, \mathbf{k})\right] \\
+ \\
+O\left(\vec{E} \cdot \nabla_{r} \psi_{n}(\mathbf{r}), \nabla_{r} \psi_{n}(\mathbf{r})^{\mathrm{T}} \nabla_{r} \psi_{n}(\mathbf{r})\right) .
\end{aligned}
$$

This is the same expression as in [5, eq. (4.7)] except that the gradient of the electrostatic potential is replaced by the gradient of the quasi-Fermi potential. By inserting (11) into the generalized drift-diffusion equation [9, eq. (4)], one finds

$$
q n \mu_{x x}^{\mathrm{eq}} E_{x}+q D_{x x}^{\mathrm{eq}} \partial_{x} n=-q n\left\langle v_{x}\right\rangle=-q n \mu_{x x}^{\mathrm{eq}} \partial_{x} \psi_{n}
$$

where $x$ is the transport direction, $E_{x}$ is the electric field in the transport direction, $\mu_{x x}^{\mathrm{eq}}$ is the component of the mobility in the transport direction from (6) evaluated in $f=f_{\mathrm{eq}}$, and $D_{x x}^{\mathrm{eq}}$ is the diffusivity in the transport direction from definition in [5, eq. (4.9)] evaluated in $f=f_{\text {eq }}$. Note that, as shown in [5, eq. (4.10)], the following relation holds between equilibrium mobility and equilibrium diffusivity

$$
\frac{k_{\mathrm{B}} T}{q} \mu_{x x}^{\mathrm{eq}}=D_{x x}^{\mathrm{eq}}
$$

which is the so-called Einstein relation. When dividing (12) by $-q n$, one finds

$$
\mu_{x x}^{\mathrm{eq}} \partial_{x} \psi_{n}=\left\langle v_{x}\right\rangle
$$

which is exactly (1). Thus, the claim is proved. If further terms of the series (11) are taken into account, (12) and (13) are not fulfilled anymore.

\section{REFERENCES}

[1] Y. Park, D. H. Navon, and T. Tang, "Monte Carlo simulation of bipolar transistors," IEEE Trans. Electron Devices, vol. ED-31, no. 12, pp. 1724 1730, Dec. 1984.

[2] P. Nguyen, D. H. Navon, and T. Tang, "Boundary conditions in regional Monte Carlo device analysis," IEEE Trans. Electron Devices, vol. ED-32, no. 4, pp. 783-787, Apr. 1985.

[3] S. Bandyopadhyay, M. Klausmeierbrown, C. Maziar, S. Datta, and M. Lundstrom, "A rigorous technique to couple Monte Carlo and drift-diffusion models for computationally efficient device simulation," IEEE Trans. Electron Devices, vol. ED-34, no. 2, pp. 392-399, Feb. 1987.

[4] H. Kosina and S. Selberherr, "A hybrid device simulator that combines Monte Carlo and drift-diffusion analysis," IEEE Trans. Comput.-Aided Design Integr. Circuits Syst., vol. 13, no. 2, pp. 201-210, Feb. 1994. 
[5] S. C. Brugger, A. Schenk, and W. Fichtner, "Moments of the inverse scattering operator of the Boltzmann equation: Theory and applications," SIAM J. Appl. Math., vol. 66, no. 4, pp. 1209-1226, 2006. [Online]. Available: http://link.aip.org/link/?SMM/66/1209/1

[6] S. Brugger and A. Schenk, "On the interpretation of local negative mobilities in nanoscale semiconductor devices," IEEE Trans. Electron Devices, vol. 54, no. 7, pp. 1766-1770, Jul. 2007.

[7] H. Gan and T. Tang, "A new method for extracting carrier mobility from Monte Carlo device simulation," IEEE Trans. Electron Devices, vol. 48, no. 2, pp. 399-401, Feb. 2001.

[8] S. C. Brugger, "Computation of semiconductor properties using moments of the inverse scattering operator of the Boltzmann equation," Ph.D. dissertation, ETH Zürich, Zürich, Switzerland, 2005. [Online]. Available: http://ecollection. ethbib.ethz.ch/show?type $=$ diss\&nr $=16376$

[9] S. C. Brugger and A. Schenk, "New one-particle Monte Carlo method for nanoscale device simulation," in Proc. Tech. NSTI Nanotechnol. Conf. Trade Show, Boston, MA, 2006, vol. 1, pp. 673-676.

[10] S. C. Brugger, A. Wirthmueller, and A. Schenk, "Quantum correction for the current-based one-particle Monte-Carlo method," in Proc. Tech. Nanotechnol. Conf. Trade Show, Santa Clara, CA, 2007, vol. 4, pp. $555-558$.

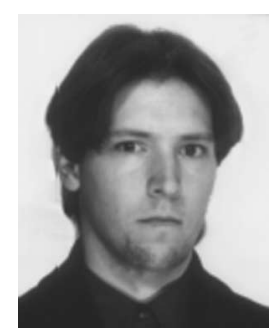

Simon C. Brugger received the Diploma in physics and the Ph.D. degree in technical sciences from the Swiss Federal Institute of Technology (ETH) Zürich, Zürich, Switzerland, in 2000 and 2005, respectively.

Since then, he has been with the Integrated Systems Laboratory, ETH in the Technology Computer-Aided Design (TCAD) research group. His professional interests include semiconductor physics, applied mathematics, and Monte Carlo simulation.

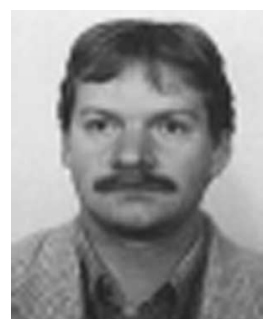

Andreas Schenk received the Dipl.-Phys. and Ph.D. degrees from Humboldt-Universität zu Berlin (HUB), Berlin, Germany, in 1981 and 1987, respectively.

From 1987 to 1991, he was working on various aspects of the physics and simulation of optoelectronic devices. In 1991, he was with the Integrated Systems Laboratory (ISL), Swiss Federal Institute of Technology (ETH) Zürich, Zürich, Switzerland, as a Senior Research/Teaching Assistant, where he qualified to give lectures at the university in 1997 for "Physics and Modeling of Microelectronic Devices." In 2004, he became Titularprofessor with the ISL. He is heading the device physics group with ISL. He is also currently with Synopsys LLC, Zürich, Switzerland. His main activities are in the following physics-based modeling of micro- and nanoelectronic devices: quantum effects in ultrashort transistors, physics and simulation of single-electron devices, many-body effects, contacts, heterojunctions, degradation effects, as well as transport processes like resonant and sequential tunneling, generation-recombination, and noise. He authored or coauthored two books and 125 papers. 